\title{
Viral Infections of the Respiratory Tract
}

\author{
George L. Kirkpatrick
}

Viral infections of the respiratory tract are responsible for large amounts of time lost from the workplace and significant morbidity and mortality in the very young and the very old. The worldwide pandemic of influenza in 1918 was alone responsible for nearly 30 million deaths in excess of those expected for influenza. Studies have demonstrated the importance of a variety of viruses, including respiratory syncytial virus and parainfluenza virus, which can both cause significant disease in high risk populations. Garibaldi and Prodine ${ }^{1}$ reported prevalence rates from $3.0 \%$ to $4.6 \%$ for all viral respiratory infections in long-term care facilities.

\section{Viruses Involved with Upper Respiratory Tract Infections}

Three studies, compared in Table 34.1, reflect similar prevalence rates for the most common upper respiratory tract viruses. A 12-month epidemiologic study of viral respiratory infections in Croatia from September 1, 1986 until August 31, 1987 studied 527 patients. ${ }^{2}$ An Indian hospital-based study conducted from 1986 to 1989 on 736 children under the age of 5 proved viral respiratory infections in $22 \%$ of the cases by using nasopharyngeal cultures. ${ }^{3}$ Finally, in a geriatric setting of institutionalized elderly, Falsey et al. found 149 nursing home residents with upper respiratory tract illnesses during a 3-month period. $^{4}$

\section{Respiratory Syncytial Virus}

Respiratory syncytial virus (RSV), a single-stranded RNA paramyxovirus, is the leading cause of pneumonia and bronchiolitis in infants and children. Two antigenically distinct groups of RSV (A and B) are recognized. Community outbreaks of RSV usually appear during the winter and spring in temperate climates. The diagnosis of RSV in the acute setting is usually obtained by viral culture of nasopharyngeal secretions. A rapid diagnostic test (Abbott test pack RSV; Directigen RSV by Becton Dickinson) employing antigen detection in nasal secretions is relatively sensitive and specific in both children and elderly.

The spectrum of illness associated with RSV is broad, ranging from mild nasal congestion to high fever and respiratory distress. RSV tends to peak during early January most years. Evidence suggests that in infants group A viruses are associated with more severe infections than group B viruses. ${ }^{5}$ Modes of spread are primarily via large droplet inoculation (requiring close contact) and self-inoculation via contaminated fomites or skin. RSV is recoverable from countertops for up to 6 hours from the time of contamination; from rubber gloves for up to 90 minutes; and from skin for up to 20 minutes. Viral shedding of RSV in infants is a prolonged process averaging 7 days. Strategies for controlling spread of RSV should be aimed at interrupting hand carriage of the virus and self-inoculation of the eyes and nose. Masks commonly employed for respiratory viruses have not been shown to be an effective measure in curtailing RSV outbreaks on pediatric wards. Hand washing is probably the single most important infection control measure for RSV.

\section{Influenza Viruses}

Influenza, often considered a benign disease today, has ravaged human populations recently enough that there are still those living who can recall the 1918 worldwide pandemic called the "Spanish flu." This particular influenza was described as beginning with what appeared to be an ordinary attack of influenza and rapidly developing into severe pneumonia. Two hours after admission the patients would have mahogany-colored spots over the cheek bones; within a few hours, cyanosis would begin spreading over the face. Shortly, death would overcome patients as they struggled for air and suffocated. The second 
Table 34.1. Prevalence of Common Upper Respiratory Tract Viruses

\begin{tabular}{|c|c|c|c|c|c|c|}
\hline \multirow[b]{2}{*}{ Virus } & \multicolumn{2}{|c|}{ Croatia study } & \multicolumn{2}{|c|}{ Indian Hospital study } & \multicolumn{2}{|c|}{ Nursing home study } \\
\hline & Patients & $\begin{array}{c}\text { Prevalence } \\
(\%)\end{array}$ & Patients & $\begin{array}{c}\text { Prevalence } \\
(\%)\end{array}$ & Patients & $\begin{array}{c}\text { Prevalence } \\
(\%)\end{array}$ \\
\hline Respiratory syncytial virus & 177 & 33.6 & 37 & 5 & 18 & 12 \\
\hline Rhinoviruses & & & & & 14 & 9.4 \\
\hline Herpes simplex & 8 & 1.3 & & & 6 & 4 \\
\hline Parainfluenza (types $1,2,3$ ) & 12 & 2.3 & 38 & 5 & 3 & 2 \\
\hline Influenza (types A and B) & 3 & 0.6 & 45 & 6 & 0 & 0 \\
\hline Adenoviruses & 40 & 7.6 & 22 & 3 & & \\
\hline Coronaviruses & & & & & & \\
\hline Enteroviruses & 18 & 4.0 & & & & \\
\hline Measles virus & & & 23 & 3 & & \\
\hline Mixed infections & 9 & 1.5 & & & 2 & 1.3 \\
\hline
\end{tabular}

documented pandemic of influenza occurred in 1957, when an outbreak of type A (antigenic determinant H2N2), from South Central China encircled the globe. Eleven years later, another epidemic emerged from eastern Asia caused by an H3N2 influenza type A. Both epidemics were associated with extensive morbidity and a marked increase in mortality, but neither was as vicious as the epidemic of 1918.

During 1977-1978 the National Health Survey estimated that 101 million acute respiratory illnesses caused by all types of respiratory viruses were medically attended in the United States. As many as 20 million cases could have been prevented by effective influenza prophylaxis. In addition to the predominant influenza virus that invades an area each season, many types, subtypes, or variants are identified during each epidemic period. These antigenically distinct viruses produce "herald waves." For several successive years, a relatively small wave of infections with an antigenically distinct virus can occur during the second half of an epidemic and herald the epidemic virus for the following year. For example, during the influenza A/Victoria epidemic of 1976, a small number of infections with influenza type $\mathrm{B} /$ Hong Kong were detected. This virus became epidemic the following year. A small wave of influenza type A (H3N2) infections during the second half of the influenza type B/Hong Kong epidemic in 1977 became epidemic during the following season. These "herald waves" are useful to epidemiologists for predicting what viral antigens should be included in each new season's vaccine. During the early stage of an epidemic, a disproportionate number of cases involve school-age children, 10 to 19 years old. Later in the epidemic, more cases are diagnosed in younger children and adults. This age shift suggests that the early spread of influenza viruses in a community is concentrated among school-age children.

Another characteristic of influenza virus is the decreased rate of infection in children living in urban areas compared to those living in rural areas. In 1974 the rate of influenza B was four times greater for children living in rural areas of Michigan than in the urban areas. Children of low income families in urban areas tend to become infected earlier in life and have milder illnesses. When these children experience intensive exposure to influenza type B during their school years, they may have immunity that is relatively more protective than that of children in the rural areas, who had less prior exposure. ${ }^{6}$

\section{Parainfluenza Viruses}

Parainfluenza is a single-strand RNA virus of which four serotypes and two subtypes are recognized (parainfluenza types $1,2,3,4 \mathrm{~A}$, and 4B). Peak activity of parainfluenza illness tends to occur in the fall and spring. These viruses can cause acute bronchitis and pneumonia in young children. Most persons have been infected with parainfluenza virus by age 5 . Immunity to parainfluenza is incomplete; and as with RSV, reinfection occurs throughout life. Parainfluenza types 1 and 2 tend to peak during the autumn of the year, whereas parainfluenza type 3 shows an increased prevalence during late spring. Adult infection results in mild upper respiratory tract symptoms, although pneumonia occasionally occurs. Outbreaks of parainfluenza types 1 and 3 have been reported from long-term care facilities. ${ }^{7}$ Illness was characterized by fever, sore throat, rhinorrhea, and cough. The rate of pneumonia was relatively high. With parainfluenza type 1 the rate was $17 \%$, and with parainfluenza type 3 the rate was $29 \%$ including several deaths.

Most studies suggest direct person-to-person transmission. Parainfluenza is stable in small-particle aerosols at the low humidity found in hospitals. However, outbreaks tend to proceed more slowly than influenza or other aerosol-spread infections. ${ }^{8}$ Infection control policies should emphasize hand washing and isolation of patients.

\section{Rhinoviruses}

Rhinovirus is the most frequent cause of upper respiratory tract infections in adults. More than 100 antigenic types have been identified, and reinfection occurs throughout life. In temperate climates, rhinovirus infection shows spring and fall peaks of activity. ${ }^{9}$ Diagnosis of a rhinovirus infection is made by viral culture of nasopharyngeal secretions. In the healthy adult, rhinovirus infection is self-limited and characterized by sneezing, rhinorrhea, and a mild sore throat. Although cough is 
common, direct viral invasion of the respiratory tract is rare. Unlike other respiratory viruses, such as influenza or adenoviruses, rhinovirus infections produce relatively minor damage to the nasal epithelium and probably no damage to the tracheal mucosa. Because rhinovirus replication is reduced at elevated body temperatures, direct invasion of the lower respiratory tract is unusual at all ages.

In one study, rhinovirus infections produced the first seasonal peak of respiratory infections in a long-term care facility. ${ }^{10}$ Seasonal peaks occur most frequently between September and November. Rhinoviruses are easily transmitted by contact with infected secretions. The most efficient modes of spread are hand-to-hand contact or direct contact with a contaminated surface followed by inoculation of the nose or conjunctiva. Rhinoviruses remain infectious for as long as 3 hours on nonporous surfaces. Transmission can be decreased by hand washing and disinfection of environmental surfaces.

\section{Coronaviruses}

Coronaviruses are single-stranded RNA viruses that have been identified as a major cause of colds in the general population. Epidemics occur during the winter and early spring. ${ }^{11}$ Infections in volunteers produced an illness similar to rhinovirus infection. Those infections produced malaise, headaches, and sore throat. Low-grade fever was present in approximately $20 \%$ of the patients. Upper respiratory tract infection is the most common result of coronavirus invasion. Coronaviruses have also been associated with exacerbations of chronic pulmonary diseases.

Diagnosis is difficult because the organism is not easy to isolate, and serology is generally not available. In a study of 11 long-term care facilities by Nicholson et al., ${ }^{12}$ patients with respiratory illnesses were analyzed for evidence of coronavirus infections. Antibodies to the two most well studied antigenic strains of coronavirus (229 E and OC43) were detected by enzyme-linked immunosorbent assay (ELISA). It was noted that illnesses were indistinguishable from RSV and influenza infections. Lower respiratory complications, such as pneumonia, occurred on one-fourth of the infected residents.

\section{Adenoviruses}

Adenoviruses are double-stranded DNA viruses, with 41 recognized serotypes that most commonly cause infections in children. Coryza, pharyngitis, pneumonia, pharyngoconjunctival fever, and epidemic keratoconjunctivitis are all attributable to adenoviruses. Transmission can occur by aerosolized droplets, fomites, and hand carriage as well as the fecal-oral route. The virus can be isolated for prolonged periods from respiratory secretions, conjunctival secretions, and stools of infected patients. The identification of adenoviruses can be confirmed by viral cultures, but the genetic heterogeneity of adenoviruses makes the information of little value. Studies have failed to show a significant incidence of infection in long-term care facilities for children or elderly patients. However, should an outbreak of adenovirus occur, strategies for control should take into account the various modes of transmission.

\section{Herpes Simplex and Measles Viruses}

Herpes simplex and measles viruses are rarely cultured from nasopharyngeal secretions. In one study ${ }^{3}$ measles virus was found in 3\% of nasopharyngeal cultures. The study also showed a case-fatality rate of $43 \%$ among patients from whom the measles virus was cultured. Similarly, herpes simplex virus showed a prevalence of $1.3 \%$ in nasopharyngeal cultures. There was no speculation about its involvement as a causative agent in the upper respiratory symptomatology.

\section{Disease Presentations}

\section{Common Cold}

The common cold, a disease of antiquity, is characterized by objective signs and subjective symptoms that are usually selflimited. Symptoms that occur with common colds include sneezing, watering of the eyes, nasal stuffiness, nasal obstruction, postnasal discharge, sore throat, hoarseness, cough, and sputum production. The common cold is a clinical diagnosis and lacks specificity because other ailments such as allergies and early effects of more serious illnesses mimic common cold symptoms.

\section{Diagnosis}

Rhinoviruses, coronaviruses, and RSV are most likely to produce the clinical findings of the common cold. These viruses are identifiable on culture. Neutralization tests with specific rabbit serum identify the rhinoviruses and the coronaviruses. RSV may be identified by immunofluorescence staining of cultured cells or ELISA studies. It is also possible to use specific immunoglobulin $\mathrm{G}$ (IgG) assays to identify rhinoviruses and coronaviruses. These tests take time, require nasopharyngeal washings or blood samples, and are an expensive way to make a definitive diagnosis.

In one study ${ }^{13} 420$ subjects were exposed to one of a variety of respiratory viruses, including rhinoviruses types 2,9 , and 14 , RSV, and coronavirus, or in a control sample simply containing saline drops. Results showed that $82 \%$ of the subjects who received intranasal drops containing virus became infected and showed signs and symptoms typical of the common cold.

\section{Management}

There are as many ways to manage the common cold as there are physicians. The use of antibiotics for the treatment of a common cold is of no proved value, as antibiotics have no effect on the viruses involved. Berkowitz and Tinkelman ${ }^{14}$ studied the effects of a nonsedating antihistamine for treating rhinitis symptoms associated with the common cold and concluded that neither the symptoms nor the signs of the common cold were alleviated to any degree. Rest, adequate hydration, and time to recover should be stressed during management of the common cold.

\section{Complications and Sequelae}

When the diagnosis of the common cold is accurate, complications and sequelae are minimal. It must be noted that those 
cases of the common cold caused by RSV are associated with significantly more fever and respiratory distress. More children than adults die as a result of RSV infections, although some deaths are reported in most studies. There are few if any deaths from rhinoviral or coronaviral common colds.

\section{Control and Prevention}

Hand washing is the most important way to prevent transmission of these viruses. With rhinoviruses and RSV, direct contact with a contaminated surface followed by inoculation of the nose or conjunctiva can result in infection. Use of masks and gloves and isolation of infected persons is the most effective way to limit the spread of cold viruses. With minimal symptoms and multiple sources of infection in the community, however, these measures are not practical.

\section{Influenza}

Influenza has one of the more characteristic sets of clinical findings. Onset is usually sudden, with shivering, sweating, headache, aching in the orbits, and general malaise and misery. Cough is often found early in the course, aggravating headaches and causing generalized aching. Onset is generally explosive with fever in adults ranging up to $102^{\circ} \mathrm{F}$. In children the fever may be higher than $102^{\circ} \mathrm{F}$, and sore throat may be an early sign. The most consistent signs are the presence of polymyalgias, weakness, and malaise.

\section{Diagnosis}

Not surprisingly, the diagnosis of influenza is more accurate during epidemics and less accurate during nonepidemic periods. Influenza in the United States usually occurs during December, January, and February. Some years, epidemics have begun earlier or continued beyond February. Successful presumptive diagnosis requires appropriate clinical symptomatology at the right time of the year and a knowledge of the pattern of influenzal illness around the world. Virologic studies, including cultures from throat swabs and nasopharyngeal washings, cells from nasopharyngeal washings stained with monoclonal antibody fluorescence stains, and complement fixation studies on paired serum samples can confirm the diagnosis. The hospital virology laboratory can suggest the most appropriate test for each case.

\section{Management}

Management of influenza is generally symptomatic. If the family physician is fairly sure that the virus in question is type A influenza, the patient may benefit greatly from the use of amantadine. Amantadine (Symmetrel) in a dose of $200 \mathrm{mg} /$ day has long been known to be excellent prophylaxis against type $\mathrm{A}$ influenza. ${ }^{15}$ It is most effective if started early in the course of the disease, although it does reduce symptoms and shortens the course of illness even if started 3 to 5 days into the disease process. The dose of amantadine is $100 \mathrm{mg}$ three times a day except for patients who have compromised renal function, for whom dosage adjustment is necessary. Because of the severity of the myalgias and headache associated with influenza, physi- cians should prescribe appropriate analgesics adequate to relieve discomfort. Aspirin and nonsteroidal antiinflammatory drugs (NSAIDs) may not suffice and a narcotic-containing product is frequently indicated. With good instructions the patient can get double benefit from codeine or hydrocodone by reducing not only the myalgias but the cough.

Amantadine is not effective for treating influenza type B. Preparing cultures or determining complement fixation titers does not provide information early enough on which to base the decision to use amantadine. Epidemiologic information is more useful.

\section{Complications and Sequelae}

Most statistical methods for assessing excess morbidity and mortality are based on an index of influenza complicated by pneumonia, which may produce an underestimation of the serious morbidity and mortality. Twice as many deaths during an influenza epidemic are attributable to ischemic heart disease than to pneumonia. ${ }^{16}$ Glezen ${ }^{16}$ studied hospital admissions with the diagnosis of acute respiratory disease during the period 1975-1976. From July 1, 1975 through June 30, 1976, a total of 3301 hospitalized patients were assessed with the admission diagnosis of acute respiratory disease. Pneumonia was the most frequent reason for admission (52.5\%). Acute bronchitis and bronchiolitis caused admission $18.9 \%$ of the time. Influenza and other respiratory disease were the cause $21.4 \%$ of the time.

Glezen also noted that the rate of hospitalization with acute respiratory disease during the influenza A/Victoria epidemic varied by age group. Infants under age 1 had a hospitalization rate of 160 per 10,000 patients. Patients between age 1 and 65 had low hospitalization rates, whereas those over 65 had a rate of 167 per 10,000 . Other studies have shown that the rate of infection is highest in the school-age population. In Glezen's study the rates of hospitalization were low in the school-age group that normally would have had the highest rates of infection.

\section{Family Issues}

Because of the considerably higher rates of infection in schoolage children, families with such children are much more likely to form clusters of influenza cases. The spread of infection goes from the child attending school to young children in the home and elderly family members.

\section{Control and Prevention}

Important epidemiologically are the facts that (1) influenza vaccine is produced each year, and (2) amantadine can be used prophylactically. The problem practitioners have using amantadine is knowing when to start and stop prophylaxis. Influenza vaccine is produced on the recommendation of the Food and Drug Administration (FDA) Vaccines and Related Biologicals Advisory Committee. Antigenic choices are based on (1) the viruses that have been seen during the previous year, (2) the viruses that are being seen in other parts of the world during the current year, and (3) the estimated antibody response in persons previously infected or vaccinated to these viruses. For example, the vaccine for the $1992-1993$ influenza season ${ }^{17}$ contained 
$\mathrm{A} / \mathrm{Texas}$, which is an $\mathrm{H} 1 \mathrm{~N} 1$ virus. It also contained $\mathrm{A} / \mathrm{Beijing}$, which is an $\mathrm{H} 3 \mathrm{~N} 2$ influenza virus, and the third viral component was a B/Panama-like influenza virus.

There are two basic strategies for the use of vaccines and chemotherapy. A current strategy is to immunize high risk groups (the elderly and children with underlying conditions including heart, pulmonary, malignant, and some metabolic diseases)..$^{18}$ Unfortunately, the level of acceptance by patients and the overall delivery of vaccines to high risk children has been consistently poor. This approach leaves most of the population unvaccinated, which produces a large "at risk" population who can be infected. Healthy persons who become infected spread their infection to nonimmunized high risk children. Another approach to the control of influenza is to immunize all schoolchildren, children in day-care, college students, military personnel, and employees of large companies. These groups have the highest susceptibility and, because of the nature of their activities, are the principal vectors of influenza virus in the community. They are also an accessible population with a structured environment that permits effective distribution of influenza vaccine. Efforts should also be directed toward immunizing as many high risk patients as possible or to start them on amantadine at the first evidence of an epidemic.

\section{Bronchitis}

\section{Diagnosis}

Bronchitis is an inflammation of the major and minor bronchial branches. It is characterized by a cough that is frequently productive of sputum, depending on the inflammatory cause. Bacterial causes of bronchitis generally produce purulent-looking sputum. Viral causes of bronchitis can cause purulent-appearing sputum but more commonly produce either clear sputum or there is a nonproductive cough. On physical examination, a patient with bronchitis has a noticeable cough but is usually normal to auscultation except for a few scattered rhonchi. Rales, dullness to percussion, egophony, and other lower respiratory findings are usually absent. Cigarette smoking, other air pollutants, and chemical exposures that cause bronchial irritation may prolong an episode of bronchitis. Systemic lupus erythematosus can be a cause of persistent bronchitis in a small number of affected patients. ${ }^{19}$

\section{Spectrum of Infection}

Studies indicate that viral causes of acute bronchitis tend to be more common with influenza (types A and B), parainfluenza of all four serotypes, and RSV. Coronavirus and adenoviruses cause bronchitis less commonly. RSV and parainfluenza viruses are found more commonly in the younger population, and coronaviruses and adenoviruses occur in older patients. Influenza causes bronchitis in all ages. Rhinovirus is the most frequent cause of upper respiratory tract infection in adults and can be a cause of coughing.

\section{Complications and Sequelae}

Liou and colleagues ${ }^{18}$ studied an influenza type B outbreak in Philadelphia from December 1985 through April 1986 and found that all patients who had positive cultures for influenza type B were under the age of 3. Fifty percent of these patients had cough and fever associated with their symptoms. Glezen, ${ }^{16}$ in a study of hospitalized patients with acute respiratory diagnoses, found acute bronchitis in $18.9 \%$. Glezen also found that adult admissions for bronchitis showed an appreciable increase only during the influenza A/Victoria epidemic, and pediatric admissions for bronchitis increased during the autumn parainfluenza types 1 and 2 epidemic and the RSV outbreak in December. Falsey et al. ${ }^{4}$ reported that in institutionalized elderly patients RSV was likely to cause bronchitis, whereas rhinovirus was more likely to cause rhinorrhea with cough as a secondary symptom. Sputum production was considerably less frequent with the rhinovirus infection than with RSV infection. Bronchitis due to parainfluenza virus is common in young children. Reinfection by parainfluenza in young, healthy adults typically presents as an upper respiratory infection including bronchitis. In the elderly, parainfluenza virus is not as common a cause of bronchitis and is much more likely to cause rhinorrhea, pharyngitis, and lower respiratory tract infections.

\section{Viral Croup}

Though croup is a frightening family experience, especially for new parents of very young children, it is a self-limited illness. Patients usually respond to breathing cool air (or in some cases warm mist in a steamed bathroom) and require little additional therapy. Those cases of croup that are not resolved by the time the family comes to the office or emergency room usually respond to racemic epinephrine administered as an aerosol. Physicians should look for the coexistence of underlying illnesses and reassure the family that croup is a manageable illness. Croup is a viral illness caused by a number of upper respiratory viral agents. In fact, during the outbreak of influenza type B in Philadelphia in 1985 and 1986, Liou et al. ${ }^{18}$ found that $4 \%$ of the children in their study less than 3 years of age had croup. Croup is common in young children and is due to parainfluenza during the fall and spring. Croup is not commonly associated with rhinovirus infections but is associated with coronavirus infections. Adenovirus is a common cause of croup in children and occurs sporadically throughout the year, being most common during the winter and spring.

\section{Bronchiolitis}

\section{Clinical Picture}

Bronchiolitis is an acute viral respiratory disease generally found in children less than 2 years old. The typical clinical presentation is an upper respiratory infection with cough that progresses to a more severe cough and tachypnea. Respirations become rapid and shallow with a prolonged expiratory phase. Because the infants are not able to breathe well, they are also unable to suck or drink and so can become dehydrated.

\section{Diagnosis}

Physical findings include intercostal retractions and nasal flaring, which may suggest pneumonia. A chest roentgenogram 
shows only hyperinflation with no infiltrates. Tight respiratory sounds (not entirely typical of wheezes found with asthma) are usually present, as are some rhonchi. Rales and dullness to percussion suggest the coexistence of pneumonia.

Bronchiolitis is most commonly caused by RSV, occurring predominantly during the winter and spring. Parainfluenza viruses, particularly types 1 and 2, can cause bronchiolitis during early winter. The most severe cases of bronchiolitis are usually caused by influenza viruses, especially type $A$. The virus involved can be identified by culture of nasopharyngeal secretions. Serologic techniques such as the ELISA and complement fixation studies are available for retrospective diagnosis, if needed.

\section{Management}

Management of bronchiolitis depends on the progression of signs and symptoms. Hospitalization may be necessary to correct hypoxemia or dehydration. If fever is significant, pneumonia must be ruled out. Cases that appear to be recurrent bronchiolitis may be asthma, even if the child is less than a year old.

Outpatient treatment is generally supportive, with careful attention to hydration. If hospitalization becomes necessary to correct hypoxemia or dehydration, treatment is focused on oxygenation, mist, and mechanically clearing the upper airway.

\section{Complications and Sequelae}

The most serious complication of bronchiolitis is respiratory failure requiring ventilatory assistance. It is best managed with continuous positive airway pressure and oxygen. Steroids and antibiotics are of questionable benefit for treating even the most severe cases of bronchiolitis. Ribavirin (Virazole) is useful as a continuous aerosol when RSV causes bronchiolitis. Ribavirin (6 $\mathrm{g}$ in $300 \mathrm{ml}$ of water) is aerosolized in a small croup tent 16 to 20 hours a day for a minimum of 3 days up to 6 days.

\section{Family Issues}

Family support is particularly important when infants require hospitalization. Many children subsequently wheeze with other viral respiratory illnesses, which alarms the family. The family needs to know that the long-term prognosis is excellent. Some research suggests that pulmonary function remains impaired in these children, especially those who have serious underlying respiratory or cardiovascular diseases.

\section{Pharyngoconjunctival Fever}

Pharyngoconjunctival fever is an upper respiratory illness that affects teenagers and adults. It manifests as pharyngitis, cough, fever, headache, myalgias, malaise, and particularly conjunctivitis. This is a syndrome caused by adenoviruses, particularly serotypes 3 and 7 . These viruses are frequently found in natural bodies of water and reservoirs.

Symptoms may be similar to those of influenza. Conjunctivitis is generally not present with influenza but is always found with pharyngoconjunctival fever and usually at an early stage. There is a spring and summer seasonal preva- lence. It can be diagnosed by viral cultures of nasopharyngeal and throat swabs or paired complement fixation antibody studies for adenovirus.

Management of pharyngoconjunctival fever is symptomatic. There is no indication for systemic antibiotic treatment or ophthalmic antibiotics. There are no long-term complications or sequelae. Recovery is generally complete within one week.

\section{Laryngitis}

There are six distinct causes of laryngitis, the most common being viral infections of the upper respiratory tract. Vocal cord tumors can cause laryngitis; allergies are a frequent cause; and strain of the vocal cords caused by long periods of loud talking produces laryngitis. A fairly frequent cause of laryngitis is hard coughing associated with either an upper or lower respiratory tract infection. The least frequent cause is a bacterial infection of the throat.

Most of the causes of laryngitis are obvious. Viral laryngitis is difficult to distinguish from the less frequent bacterial laryngitis that might require antibiotic treatment.

Children over age 2 and adults rarely have significant swelling of the throat that would put them at risk of airway obstruction. Children under age 2 are more likely to develop airway obstruction. Viral causes of laryngitis include the parainfluenza viruses, rhinoviruses, adenoviruses, and the influenza viruses.

Voice rest makes the greatest impact on recovery. Patients who are able to gargle with warm, very weak sale water solution sometimes find it soothing. Being a fairly common complaint in the family physician's office, patients should be told that it is not a serious disease and that adequate time to recover is the only therapy in most cases.

\section{Viral Respiratory Tract Infections in Very Young and Old Patients}

Patients below age 2 present some special problems. Perhaps as many as two-thirds of pediatric emergency room visits for respiratory infections are inappropriate.

Mayefsky et al. ${ }^{20}$ studied the pediatric emergency department at Cook County Hospital in Chicago, where 60,000 children between the ages of birth and 16 years of age were seen annually. Mayefsky et al. concluded that parents using the emergency department for evaluation of their children's colds have reasonable concerns for the health of their children. More than $50 \%$ of the parents needed only reassurance that their child was not seriously ill.

Mullooly and Barker ${ }^{21}$ studied the impact of type A influenza on children at the Kaiser-Permanente Medical Care Program in Oregon from 1968 to 1973 . They compared excess morbidity to excess rates of hospitalization for influenza-related conditions. Excess hospitalization rates for the under-age-4 group during influenza epidemics were three to five times higher than the rates for children over age 4.

The study by Sugaya and Nerome ${ }^{22}$ in Japan during the winter of 1989 and spring of 1990, where the epidemics of influenza type A H3N2 and influenza type $B$ viruses were 
Table 34.2. Patterns of Viral Illness in Children and Elderly Patients

\begin{tabular}{|c|c|c|c|}
\hline \multirow[b]{2}{*}{ Virus } & \multicolumn{3}{|c|}{ Signs and symptoms } \\
\hline & Young children & Adults & Elderly \\
\hline $\begin{array}{l}\text { Respiratory syncytial } \\
\text { virus }\end{array}$ & $\begin{array}{l}\text { Wheezing, bronchiolitis } \\
\text { pneumonia, bronchitis }\end{array}$ & Nasal congestion and cough & $\begin{array}{l}\text { Nasal congestion, cough, } \\
\text { fever, pneumonia, } \\
\text { wheezing, bronchitis }\end{array}$ \\
\hline Influenza & $\begin{array}{l}\text { Sore throat, high fever, } \\
\text { myalgias, bronchitis, croup, } \\
\text { bronchiolitis, rhinorrhea, } \\
\text { otitis media }\end{array}$ & $\begin{array}{l}\text { Fever, headache, myalgias, } \\
\text { malaise, cough, weakness, } \\
\text { bronchitis, laryngitis }\end{array}$ & $\begin{array}{l}\text { Bronchitis, low grade fever, } \\
\text { sore throat, pneumonia }\end{array}$ \\
\hline Parainfluenza & $\begin{array}{l}\text { Croup, bronchitis, pneumonia, } \\
\text { sore throat, bronchiolitis }\end{array}$ & Common cold, laryngitis & $\begin{array}{l}\text { Rhinorrhea, sore throat, } \\
\text { cough, pneumonia, fever }\end{array}$ \\
\hline Rhinoviruses & Sore throat, rhinorrhea & $\begin{array}{l}\text { Rhinorrhea, sneezing, cough, } \\
\text { sore throat, laryngitis }\end{array}$ & Rhinorrhea, cough, sneezing \\
\hline Coronaviruses & Croup, sore throat & $\begin{array}{l}\text { Common cold, malaise, } \\
\text { headache, sore throat, low } \\
\text { grade fever }\end{array}$ & $\begin{array}{l}\text { Exacerbation of chronic } \\
\text { pulmonary disease, } \\
\text { pneumonia, bronchitis }\end{array}$ \\
\hline Adenoviruses & Croup, sore throat & $\begin{array}{l}\text { Coryza, sore throat, } \\
\text { pneumonia, pharyngo- } \\
\text { conjunctival fever, } \\
\text { keratoconjunctivitis, } \\
\text { laryngitis }\end{array}$ & Bronchitis rarely \\
\hline
\end{tabular}

severe, showed a remarkable increase in the number of pediatric admissions.

Although most respiratory virus infections in children appear to be self-limited and without complications or sequelae, mortality statistics in 1976 showed that infections of the respiratory tract rank third among the leading causes of death in infants 28 days to 1 year of age and second in children between 1 and 4 years of age. Table 34.2 details the patterns of viral illness found in young children, adults, and elderly patients.

The institutionalized elderly represent a subgroup of older people who are prone to excess morbidity and mortality from respiratory tract infections. Each year many elderly persons living in long term care facilities become ill with respiratory illnesses that are mistakenly attributed to bacterial pneumonia or influenza. The respiratory tract viruses seen in Table 34.2 (particularly RSV, parainfluenza virus, and influenza virus) are a significant cause of disease in this high risk population. Large epidemiologic studies have found a predominance of noninfluenza agents responsible for respiratory tract disease. RSV ranks second to influenza as the most common cause of serious viral respiratory infections in long term care facility patients. A significant number of reported deaths are of cardiovascular origin, related to the viral infection rather to pneumonia. ${ }^{16}$ The pattern of reported outbreaks of RSV in a long term care facility is usually a steady trickle of cases over several months-distinctly different from outbreaks of influenza, which tend to be explosive. Parainfluenza virus is a common cause of croup and bronchitis in young children; however, because full immunity does not develop, reinfection is common in the older population. In the institutionalized elderly, parainfluenza presents as rhinorrhea, pharyngitis, cough, and pneumonia.

\section{References}

1. Garibaldi RA, Brodine S. Infections among patients in nursing homes: policies, prevalence, and problems. N Engl J Med 1982;305:731-5.

2. Mlinaric G. Epidemiological picture of respiratory viral infections in Croatia. Acta Med Iugosl 1991;45:203-11.

3. Jain A. An Indian hospital study of viral causes of acute respiratory infection in children. J Med Microbiol 1991;35:219-23.

4. Falsey AR, Treanor JJ. Viral respiratory infections in the institutionalized elderly; clinical and epidemiology findings. J Am Geriatr Soc 1992;40:115-19.

5. McConnochie KM, Hall CB. Variation in severity of respiratory syncytial viruses with subtype. J Pediatr 1990;117:52-58.

6. Glezen WP. Consideration of the risk of influenza in children and indications for prophylaxis. Rev Infect Dis 1980;2:408-20.

7. Public Health Laboratory Service Communicable Disease Surveillance Center. Parainfluenza infections in the elderly 1976-1982. BMJ 1983;287:1619.

8. Graman PS, Hall CB. Epidemiology and control of nosocomial viral infections. Infect Dis Clin North Am 1989;3:815-41.

9. Gwaltney JM, Mandell GL, Douglas RG Jr. Principles and practices of infectious diseases, 3rd ed. New York: Churchill Livingstone, 1989:1399-1404.

10. Grose PA, Rodstein M. Epidemiology of acute respiratory illness during an influenza outbreak in a nursing home: a prospective study. Arch Intern Med 1988;148:559-61. 
11. Larsen HE, Reed JE. Isolation of rhinoviruses and coronaviruses from 38 colds in adults. J Med Virol 1980;5:221-9.

12. Nicholson KG, Baker DJ. Acute upper respiratory tract illness and influenza immunizations in homes for the elderly. Epidemiol Infect 1990;105:609-18.

13. Cohen S, Tyrrell DAJ. Psychological stress and susceptibility to the common cold. N Engl J Med 1991;29:606-11.

14. Berkowitz RB, Tinkelman DG. Evaluation of oral terfenadine for treatment of the common cold. Ann Allergy 1991;67:593-7.

15. Kobayashi JM. Control of influenza A outbreaks in nursing homes: amantadine as an adjunct to vaccine-Washington, 1989-90. MMWR 1991;40:841-4.

16. Glezen WP. Serious morbidity and mortality associated with influenza epidemics. Epidemiol Rev 1982;4:25-43.
17. Nerome K, Chakraverty M. Update: influenza activity-United States and worldwide, and composition of the 1992-1993 influenza vaccine. MMWR 1992;41:316-7.

18. Liou YS, Barbour SD, Bell LM. Children hospitalized with influenza B infection. Pediatr Infect Dis J 1987;6:541-3.

19. Raz E, Bursztyn M. Severe recurrent lupus laryngitis. Am J Med 1992;92:109-10.

20. Mayefsky JH, El-Shinaway Y. Families who seek care for the common cold in a pediatric emergency department. J Pediatr 1991; 119:933-4.

21. Mullooly JP, Barker WH. Impact of type A influenza on children: a retrospective study. Am J Public Health 1982;72:1008-16.

22. Sugaya N, Nerome K. Impact of influenza virus infection as a cause of pediatric hospitalization. J Infect Dis 1992;165:373-5. 